\title{
Validity of serum leukotriene B4 as a marker of decompensated liver cirrhosis in chronic HCV patients: leukotriene B4 in decompensated liver cirrhosis
}

\author{
Ayman El-Shayeb ${ }^{1}$, Sawsan El-Mallah¹, Akram Deghady ${ }^{2}$, Samar El-Hewely ${ }^{1}$ and Doaa Elwazzan ${ }^{1 *}$ (1)
}

\begin{abstract}
Background: Liver cirrhosis is divided into two stages: compensated stage and another advanced stage which includes both decompensated cirrhosis and acute-on-chronic liver failure. The immune system is of major importance in cirrhosis pathophysiology. Sustained bacterial translocation from the intestine to systemic circulation causes a chronic systemic inflammatory syndrome. Inflammatory markers are emerging in the scope of cirrhosis and its complications due to their deleterious effects on disease progression and prognosis. The aim of the study was to assess the value of leukotriene B4 (LTB4) as a marker of decompensated cirrhosis and to evaluate its relation to disease progression and complications.

Results: The current study was conducted on 80 candidates in the Alexandria Main University Hospital, Tropical Medicine Department; they were categorized into three groups: group I $(n=30)$ compensated cirrhotic patients, group II ( $n=30)$ decompensated cirrhotic patients, and group III $(n=20)$ apparently healthy individuals. Serum LTB4 was measured by ELISA. LTB4 showed statistically significant higher values in the decompensated group than the compensated group $(p=0.007)$ and the control group $(p=0.002)$. However, there was no statistically significant difference between the compensated group and the controls $(p=0.510)$. LTB4 correlated positively with the ChildPugh score $(p=0.003)$. Moreover, it correlated positively with the MELD-Na score $(p=0.012)$. There was a significant correlation between the degree of ascites and serum LTB4 $(p<0.001)$. However, there was no significant correlation between hepatic encephalopathy and LTB4 $(P>0.204)$.
\end{abstract}

Conclusion: Serum LTB4 could be used as a sensitive biomarker of decompensation in cirrhotic patients.

Keywords: Hepatitis C virus, Liver cirrhosis, Bacterial translocation, Leukotriene B4

\section{Background}

Chronic HCV infection is often asymptomatic and ultimately leads to cirrhosis [1]. The clinical course of cirrhosis is divided into two stages: compensated and decompensated [2]. Progression of a compensated stage to a decompensated one has a significantly negative effect

\footnotetext{
*Correspondence: d.elwazzan@gmail.com

${ }^{1}$ Tropical Medicine Department, Faculty of Medicine, Alexandria University, Alexandria, Egypt

Full list of author information is available at the end of the article
}

on the prognosis and is related to an abrupt decrease in survival probability to less than $3-5$ years [3].

Our concepts on the pathophysiology of cirrhosis progression have undergone major changes and the axis formed by the bowel, liver, and immune system became of major importance [4]. Sustained bacterial translocation from the intestinal lumen to the systemic circulation and organs triggers the innate immune system and results in a chronic systemic inflammatory syndrome which may be a mechanism for decompensation and multiorgan dysfunction [5]. 
LTB4 is a pro-inflammatory compound that displays strong chemoattractant properties. Increased LTB4 has been found in lots of inflammatory and allergic diseases and levels correlate with disease activity in some of these [6]. However, leukotriene B4 role in decompensated cirrhosis has not been studied in depth.

\section{Aim of the work}

The aim of this study was to assess the value of LTB4 as a marker of decompensated cirrhosis and to study its relation to complications and disease progression.

\section{Subjects}

This study was conducted on 80 candidates in the Alexandria Main University Hospital, Tropical Medicine Department; subjects were divided into three groups: group I $(n=30)$ compensated cirrhotic patients, group II $(n=30)$ decompensated cirrhotic patients, and group III $(n=20)$ apparently healthy individuals. The age of the candidates ranged between 21 and 79 years old.

\section{Exclusion criteria}

Patients with concurrent allergic diseases, diabetes mellitus, obesity, malignancies, and history of anti-inflammatory and immunosuppressive drugs were excluded from the study.

\section{Methods}

All patients were subjected to thorough history taking and proper clinical examination. Laboratory investigations included complete blood count, liver function tests, renal function tests, erythrocyte sedimentation rate and $C$ reactive protein (CRP), viral hepatitis markers $B$ and $C$, polymerase chain reaction test for HCV RNA, and serum alpha-fetoprotein. Triceps fold thickness and basal metabolic index were estimated in addition to Child-Pugh classification and MELD score calculation. Serum LTB4 was measured by the ELISA kit named Human Leukotriene B4 (LTB4) ELISA kit. Ultrasound abdomen and triphasic $\mathrm{CT}$ scan were done for patients with ultrasound positive focal hepatic lesions.

Data were analyzed using the IBM SPSS software package version 20.0. The used statistical tests were chi-square test, Monte Carlo correction, Student's $t$ test, $F$-test (ANOVA), Mann-Whitney test, Kruskal-Wallis test, and Spearman's coefficient. Receiver operating characteristic curve (ROC (was generated by plotting sensitivity (TP) on $Y$-axis versus 1 -specificity (FP) on $X$-axis at different cutoff values. The area under the ROC curve denotes the diagnostic performance of the test. Area more than 50\% gives acceptable performance and area about $100 \%$ is the best performance for the test.

\section{Ethical approval}

The ethical committee of the Faculty of Medicine, Alexandria University, approved this study. Written informed consent was obtained from all patients.

\section{Results}

This study was conducted on 80 candidates in the Alexandria Main University Hospital, Tropical Medicine Department; subjects were divided into three groups.

Regarding their demographic data, see Table 1.

Group I included 30 compensated cirrhotic patients. They were $63.3 \%$ males and $36.7 \%$ females. Their mean age was $48.0 \pm 11.06$ years old. All the patients were $\mathrm{HCV}$ positive.

Group II included 30 decompensated cirrhotic patients. They were $50 \%$ females and $50 \%$ males. Their mean age was $55.47 \pm 12.62$ years old. All the patients were $\mathrm{HCV}$ positive.

Group III control group included 20 apparently healthy individuals. They were $35.0 \%$ males and $65 \%$ females. Their mean age was $55.47 \pm 12.62$ years old.

Regarding LTB4 results, in the control group, it ranged $6.21-47.45 \mathrm{ng} / \mathrm{L}$ with a mean of $19.83 \pm 13.17 \mathrm{ng} / \mathrm{L}$. In compensated cirrhosis group, it ranged 9.11-58.73 ng/L with a mean of 9.11-58.73 ng/L. On the other hand, LTB4 in the decompensated cirrhosis group ranged between 13.62 and $346.1 \mathrm{ng} / \mathrm{L}$ with a mean of $47.34 \pm 65.18 \mathrm{ng} / \mathrm{L}$. LTB4 showed a statistically significantly higher value in the decompensated cirrhosis group than the compensated cirrhosis group $(p=0.007)$ and the control group $(p$ $=0.002$ ). However, there was no statistically significant difference between the compensated cirrhosis group and the control groups with $p=0.510$ (Table 1, Fig. 1). Going through lab results (Table 1), bilirubin was statistically significantly higher in group II than group I $(p<0.001)$. Albumin was statistically significantly lower in group II than group I $(p<0.001)$. INR was statistically significantly higher in group II than group I $(p<0.001)$.

In the current work, we assessed serum creatinine and serum sodium in the studied groups. Creatinine was statistically significantly higher in group II than group I with $(p=0.007)$. There was a positive correlation between serum level of creatinine and LTB4 where $p=0.035$. As regards serum sodium, it was statistically significantly lower in group II than group I and III ( $p=$ 0.001 ); however, there was no statistically significant difference between group I and III ( $p=0.798)$. There was a statistically significant correlation between serum level of sodium and LTB4 where $p=0.040$. LTB4 showed higher values in patients with lower sodium levels.

Regarding serum CRP, it was statistically significantly higher in the patients than in the control group. 
Table 1 Comparison between the three studied groups $(n=80)$ according to demographic data and other different parameters

\begin{tabular}{|c|c|c|c|c|c|}
\hline & Group I $(n=30)$ & Group II $(n=30)$ & Group III $(n=20)$ & Test of Sig. & $P$ \\
\hline \multicolumn{6}{|l|}{ Gender } \\
\hline Male & 19 & 15 & 7 & \multirow[t]{2}{*}{$x^{2}=3.886$} & \multirow[t]{2}{*}{0.143} \\
\hline Female & 11 & 15 & 13 & & \\
\hline \multicolumn{6}{|l|}{ Age (years) } \\
\hline Min-Max & $28.0-70.0$ & $36.0-79.0$ & $21.0-45.0$ & \multirow[t]{3}{*}{$F=39.150^{*}$} & \multirow[t]{3}{*}{$<0.001^{*}$} \\
\hline Mean \pm SD & $48.0 \pm 11.06$ & $55.47 \pm 12.62$ & $28.60 \pm 5.36$ & & \\
\hline Median (IQR) & $45.50(39.0-57.0)$ & $54.0(45.0-67.0)$ & $28.0(25.50-31.0)$ & & \\
\hline \multicolumn{6}{|l|}{ Degree of ascites } \\
\hline Mild & - & 9 & - & 30 & \multirow[t]{3}{*}{ - } \\
\hline Moderate & - & 9 & - & 30 & \\
\hline Massive & - & 12 & - & 40 & \\
\hline Hepatic encephalopathy & - & 17 & - & $x^{2}=23.721^{*}$ & $<0.001^{*}$ \\
\hline \multicolumn{6}{|l|}{ Total bilirubin (mg/dl) } \\
\hline Min.-Max. & $0.20-1.40$ & $0.50-9.0$ & $0.80-1.20$ & $H=29.980^{*}$ & $<0.001^{*}$ \\
\hline \multicolumn{6}{|l|}{ Serum albumin (g/dl) } \\
\hline Min.-Max. & $3.50-4.30$ & $1.60-3.0$ & $4.0-5.50$ & $F=245.85^{*}$ & $<0.001^{*}$ \\
\hline \multicolumn{6}{|l|}{ INR } \\
\hline Min.-Max. & $0.80-1.50$ & $1.10-2.50$ & $0.90-1.40$ & $F=45.043^{*}$ & $<0.001^{*}$ \\
\hline \multicolumn{6}{|l|}{ CRP } \\
\hline Min.-Max. & $10.0-18.0$ & $24.0-50.0$ & $1.0-5.0$ & $H=69.618^{*}$ & $<0.001^{*}$ \\
\hline \multicolumn{6}{|l|}{ Child-Pugh Classification } \\
\hline A & 30 & 0 & NA & $x^{2}=60.00^{*}$ & $<0.001^{*}$ \\
\hline B & 0 & 15 & & & \\
\hline C & 0 & 15 & & & \\
\hline \multicolumn{6}{|l|}{ MELD/Na score } \\
\hline Min-Max & $6.0-18.0$ & $10.0-30.0$ & NA & $t=10.592^{*}$ & $<0.001^{*}$ \\
\hline Mean \pm SD & $10.43 \pm 3.07$ & $20.23 \pm 4.03$ & & & \\
\hline Median (IQR) & $10.0(9.0-12.0)$ & $20.0(18.0-22.0)$ & & & \\
\hline \multicolumn{6}{|c|}{ Serum Leukotriene B4 (ng/L) } \\
\hline Min.-Max & $9.11-58.73$ & $13.62-346.1$ & $6.21-47.45$ & $H=11.517^{*}$ & $0.003^{*}$ \\
\hline Mean $\pm S D$ & $21.12 \pm 11.0$ & $47.34 \pm 65.18$ & $19.83 \pm 13.17$ & & \\
\hline Median (IQR) & $17.90(12.63-28.69)$ & 24.27(19.59-39.35) & $13.11(9.13-27.23)$ & & \\
\hline
\end{tabular}

Group I: Compensated liver cirrhosis due to chronic HCV infection

Group II: Decompensated liver cirrhosis due to chronic HCV infection

Group III: Healthy controls

INR International normalization ratio, CRP C-reactive protein, $X^{2}$ Chi-square test, $H$ Kruskal-Wallis test, pairwise comparison bet. the 2 groups were done using post hoc test (Dunn's for multiple comparisons test), $F$ ANOVA test, pairwise comparison bet. the 2 groups were done using post hoc test (Tukey), $t$ Student's $t$ test, $p p$ value for comparing between the studied groups

*Statistically significant at $p \leq 0.05$

Moreover, it was statistically significantly higher in group II than group I $(p=0.001)$. There was a statistically significant positive correlation between CRP and LTB4 $(p<0.006)$ (Table 1, Fig. 2).

All patients in the decompensated cirrhosis group were suffering from different degrees of ascites. Twelve out of thirty patients presented with a massive degree of ascites. The rest of the patients, half of them, had moderate ascites and the other half had a mild degree of ascites assessed by ultrasound (Table 1 ). The relation between LTB4 and degree of ascites was assessed and results showed a positive correlation between LTB4 and the degree of ascites $(p<0.001)$. Serum LTB4 levels in patients with massive ascites ranged 32.39$346.1 \mathrm{ng} / \mathrm{L}$. It was lower in patients with moderate ascites ranging $20.54-27.81 \mathrm{ng} / \mathrm{L}$. The lowest values were in patients with a mild degree of ascites ranging $13.62-19.88 \mathrm{ng} / \mathrm{L}$. 


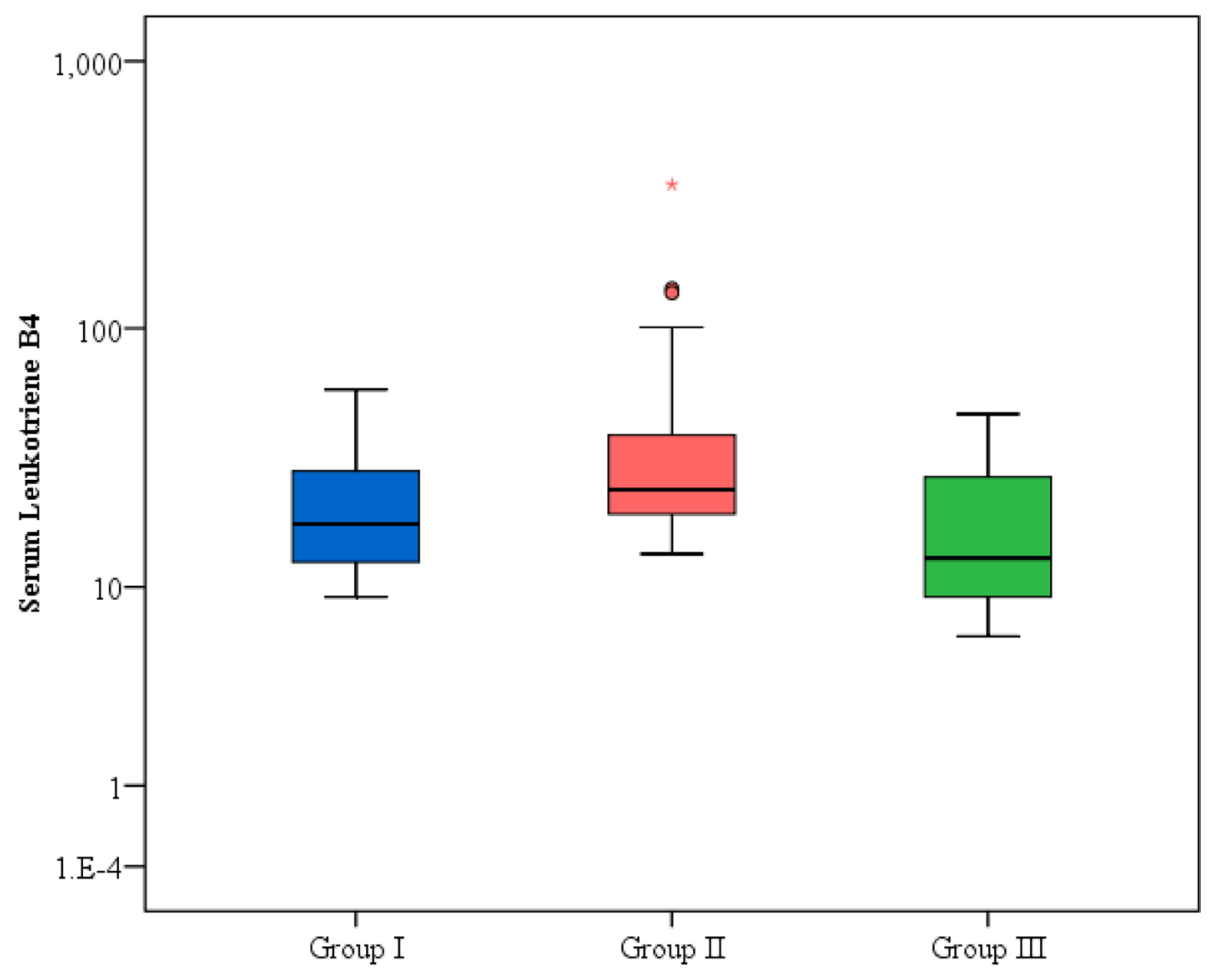

Fig. 1 Comparison between the three studied groups $(n=80)$ according to serum LTB4

Seventeen out of thirty decompensated patients were suffering from hepatic encephalopathy (Table 1). In these patients, LTB4 ranged 13.62-346.1 ng/L compared to patients without hepatic encephalopathy where LTB4 ranged 9.11-140.8 ng/L. There was no significant correlation between hepatic encephalopathy and LTB4 $(p>0.204)$.

As regards the Child-Pugh classification, in group I, all patients were Child A class. Group II included 15 patients with Child score B and 15 patients with Child score $\mathrm{C}$ (Table 1 ). There was a statistically significant difference between groups I and II $(p<0.001)$. The Child classification showed a statistically significant correlation with LTB4 $(p=0.003)$ (Fig. 3).

MELD/Na score calculation was done; it ranged 6.018.0 in the compensated cirrhosis group. On the other hand, MELD/Na score ranged 10.0-30 in the decompensated group (Table 1 ). It was statistically significantly higher in decompensated cirrhosis group than in compensated group $(p<0.001)$. The relation between LTB4 and Meld/Na score showed a statistically significant correlation $(p<0.012)$ (Fig. 4).

ROC curve was done to estimate the cutoff point of LTB4 to discriminate compensated cirrhosis from decompensated cirrhosis. The ROC curve for Serum LTB4 was significant ( $\mathrm{p} 0.002 \%$ ) and showed that the cutoff level of LTB4 discriminating decompensated patients from compensated patients was $>16.2 \mathrm{ng} / \mathrm{L}$, with a sensitivity of $93.33 \%$, specificity of $43.33 \%$, positive predictive value of $62.2 \%$, and negative predictive value of $86.7 \%$ with a diagnostic accuracy of $66.7 \%$. (Table 2, Fig. 5)

\section{Discussion}

There is now much evidence that inflammatory mediators have direct deleterious effects in the pathophysiology of liver cirrhosis and its complications [7]. Several studies have shown several circulatory chemokines involved in leukocyte migration and chemotaxis to be increased in decompensated cirrhotic patients, which correlated with survival [8].

Leukotrienes are potent biological mediators implicated in lots of disease processes. LTB4 is a pro-inflammatory compound that displays strong chemoattractant properties. Increased LTB4 has been found in lots of inflammatory and allergic diseases and levels correlate with disease activity in some of these [9].

In the current study, serum LTB4 value was elevated with worsening severity of liver disease measured by Child-Pugh classification and MELD/Na score. LTB4 was significantly higher in decompensated cirrhosis group 


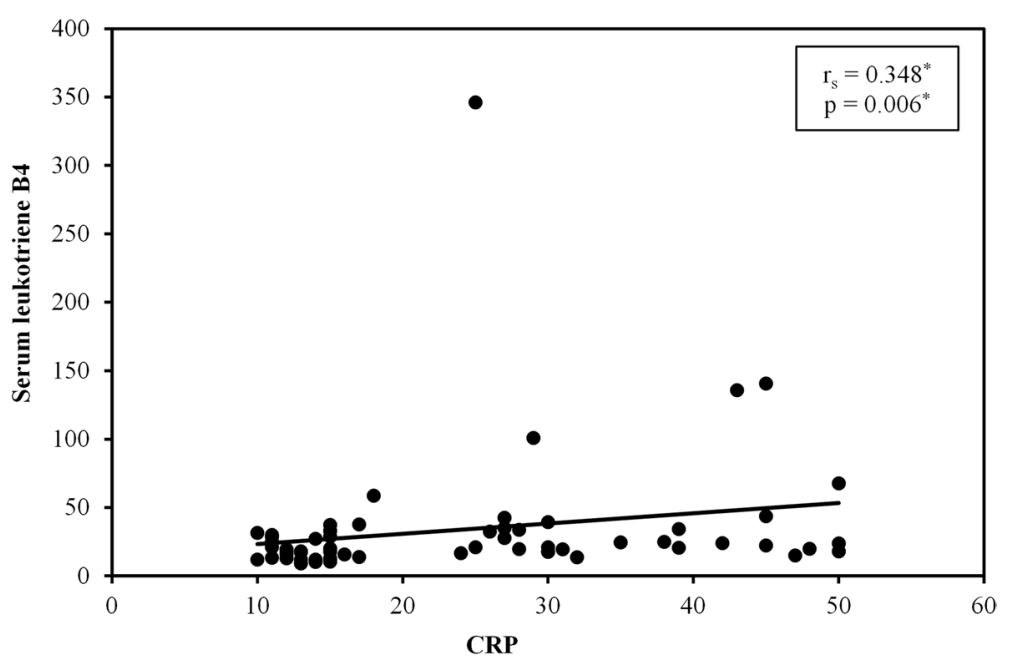

Fig. 2 Correlation between serum leukotriene B4 and CRP in total cases $(n=60)$

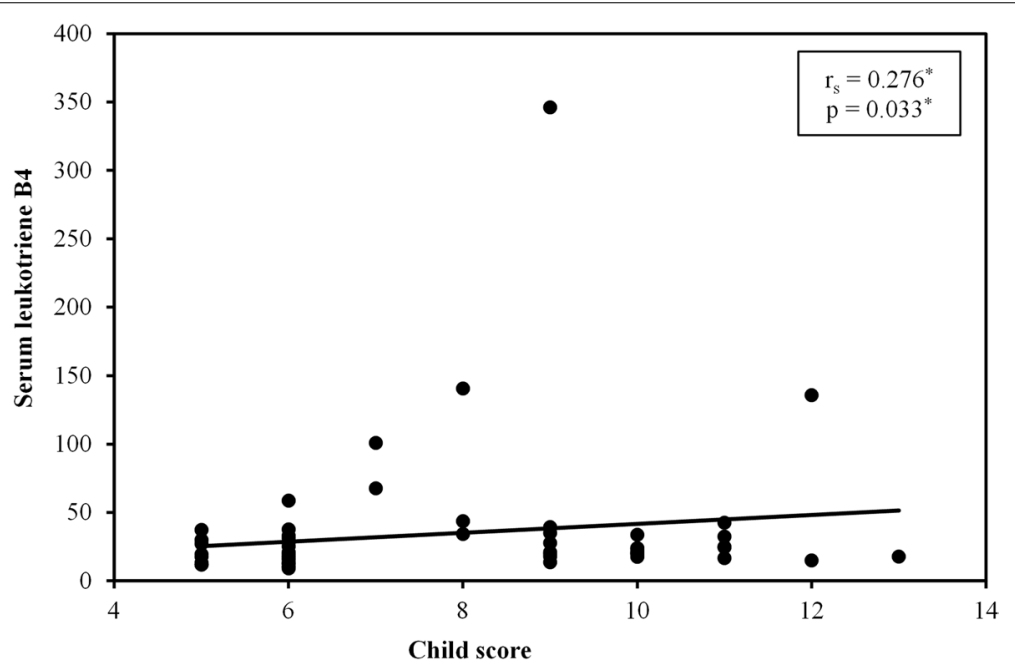

Fig. 3 Correlation between LTB4 and Child Score in cirrhotic patients $(n=60)$

than the compensated group and controls $(p=0.003)$. However, there was no significant difference between compensated cirrhosis group and controls $(p=0.510)$. LTB4 showed a statistically significant correlation with Child class $(p=0.003)$. Moreover, LTB4 values showed a significant positive correlation with MELD/Na score $(p=$ 0.012). This proves the idea of systemic inflammation in cirrhosis which may be a mechanism for decompensation and multiorgan dysfunction.

Our results showed that LTB4 cutoff value to discriminate compensated cirrhosis from decompensated cirrhosis was $>16.2 \mathrm{ng} / \mathrm{L}$ with a sensitivity of $93.33 \%$ and specificity of $43.33 \%$.
In addition to our study, Becares et al. study [10], quantified serum LTB4 in a cohort of 26 patients with cirrhosis divided into 3 groups: group I healthy volunteers $(n=$ $5)$, group II decompensated stable patients $(n=11)$, and group III ACLF $(n=10)$. Becares et al. study [10] concluded that ACLF patients display significantly higher neutrophil recruitment to a site of inflammation than healthy volunteers in addition to increased plasma levels of the chemotactic LTB4 according to cirrhosis disease severity which was compatible with our findings.

Another study discussing the value of LTB4 in chronic liver disease is Asano et al's study [11], which measured LTB4 using peripheral blood mononuclear cells (PBMC) 


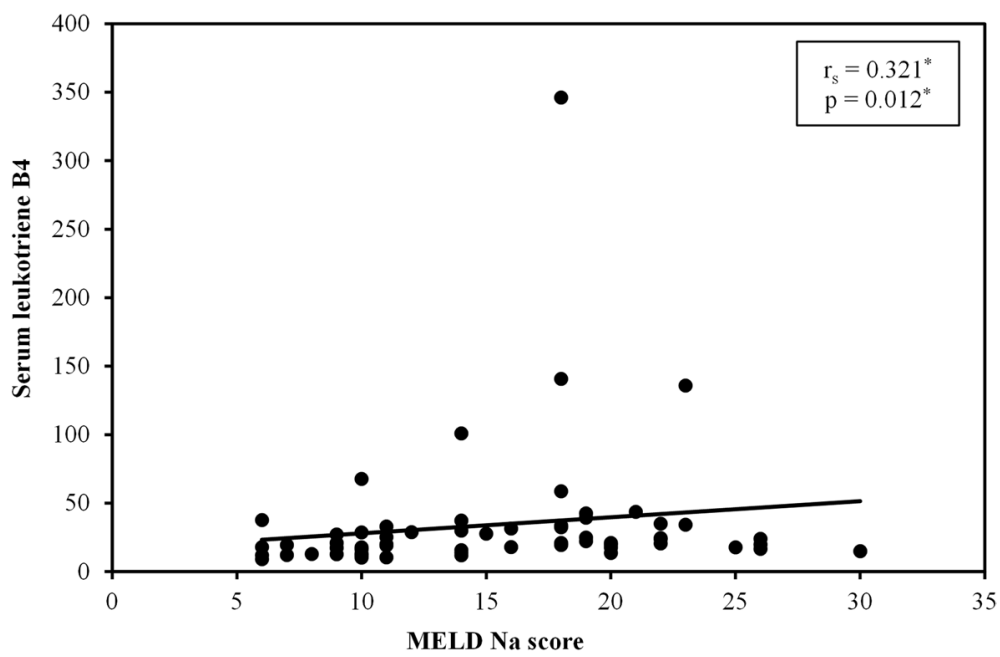

Fig. 4 Correlation between LTB4 and MELD/Na score in cirrhotic patients $(n=60)$

Table 2 Agreement (sensitivity, specificity) for LTB4 to diagnose decompensated cirrhosis group $(n=30)$ from compensated cirrhosis group $(n=30)$

\begin{tabular}{llllllllll}
\hline & AUC & $\boldsymbol{P}$ & $\mathbf{9 5 \%}$ C.I & Cut off $^{\mathrm{a}}$ & Sensitivity $\%$ & Specificity \% & PPV \% & NPV \% & Accuracy of the test \% \\
\hline Serum leukotriene B4 & 0.728 & $0.002^{*}$ & $0.601-0.855$ & $>16.2$ & 93.33 & 43.33 & 62.2 & 86.7 & 66.7 \\
\hline
\end{tabular}

AUC Area under a curve, $p$ value Probability value, CI Confidence Intervals, NPV Negative predictive value, PPV Positive predictive value

*Statistically significant at $p \leq 0.05$

${ }^{\text {a }}$ Cutoff was chosen according to the Youden index

in patients with fulminant hepatitis $(\mathrm{FH})$ and other liver diseases. The production rate of LTB4 was elevated in 16 of $17 \mathrm{FH}$ patients. However, in discordance to our study, LTB4 was below the detectable level in patients with chronic hepatitis. Asano et al. concluded that enhanced LTB4 production may indicate the primed state of PBMC and their role in the development of massive liver necrosis and of other organ failures in $\mathrm{FH}$.

Serum sodium was measured and results showed statistically lower values in Child B and C than Child A patients $(p<0.001)$ and controls $(p<0.001)$; however, there was no statistically significant difference between group I and III ( $p=0.798)$. This was in agreement with James et al.s study [12], who evaluated serum sodium among 97 decompensated patients, 42 had serum sodium levels $\geq 136 \mathrm{mEq} / \mathrm{L}$, while 32 had serum sodium levels between 131 and $135 \mathrm{mEq} / \mathrm{L}$ and 23 patients had serum sodium level $\leq 130 \mathrm{mEq} / \mathrm{L}$. Serum sodium levels were associated strongly with the severity of liver disease as assessed by Child-Pugh and MELD score. Patients with serum sodium levels $\leq 130 \mathrm{mEq} / \mathrm{L}$ had increased mortality (30.4\% with $p=0.002)$. In our study, serum sodium showed a significant negative correlation with LTB4 ( $p=$ $\left.0.040^{*}\right)$.
Regarding CRP, it was statistically significantly higher in Child B and C patients $(p<0.001)$ than Child A patients. This was compatible to Lai et al's study [13] where patients with class $\mathrm{B}$ and $\mathrm{C}$ scores showed the highest level of high-sensitivity $\mathrm{C}$-reactive protein when compared with class A patients or healthy subjects. Lai et al. [13] aimed to assess the association of liver cirrhosis with increased inflammatory responses and the relation between CRP and the severity of the liver disease. In our study, LTB4 correlated positively with CRP values with $p$ $=0.006$.

LTB4 correlated positively with the degree of ascites (mild - moderate - massive) with $p<0.001$. LTB4 relation to ascites was studied in Ouwendijk et al. study [14]; they collected ascitic fluid from six patients with liver cirrhosis and isolated macrophages that were labeled with 14C-arachidonic acid. After stimulation of these cells, the main substances formed were LTB4 and LTC4. This suggests that human peritoneal macrophages are capable of producing LTs and prostaglandins. Moreover, it supports the idea that the production of these substances might play a role in some of the complications of patients with liver cirrhosis and ascites. 


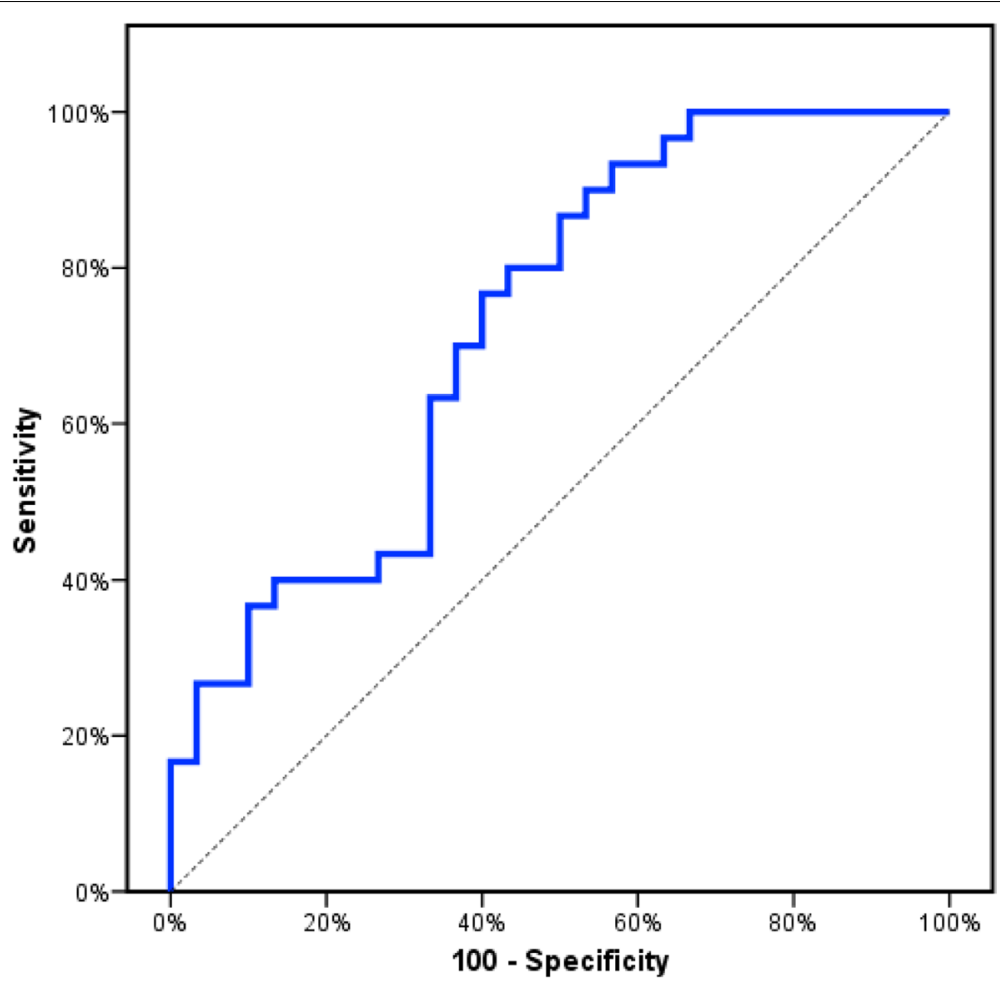

Fig. 5 ROC curve for LTB4 to discriminate compensated cirrhosis patients $(n=30)$ from decompensated cirrhosis patients $(n=30)$

Other leukotrienes studied in cirrhosis and its related complications are cysteinyl leukotrienes and their metabolite leukotriene E4 (LTE4). According to Moore et al's study [15], urinary LTE4 was elevated in patients with HRS compared with normal controls and with renal failure alone. Urinary LTE4 was also elevated in subjects with decompensated liver disease; it showed high results in cirrhosis with ascites and even higher results in severe hepatocellular dysfunction; however, it was normal in compensated liver disease. They concluded that LTE4 production is increased in HRS and that this may be one of the factors involved in its pathogenesis.

\section{Conclusions}

Serum LTB4 could be used as a sensitive biomarker of decompensation in cirrhotic patients. Furthermore, the significant correlation of serum LTB4 with Child-Pugh class, MELD/Na score, and CRP could suggest that it can be used as a tool to follow up the clinical outcomes of patients with liver cirrhosis and prioritize listing for liver transplantation.

\section{Abbreviations}

LTB4: Leukotriene B4; MELD: Model for end-stage liver disease; HCV: Hepatitis C virus; CRP: C-reactive protein; ACLF: Acute on chronic liver failure; PBMC: Peripheral blood mononuclear cells; LTE4: Leukotriene E4.

\section{Acknowledgements}

The authors would like to thank all patients for their participation in this research.

\section{Authors' contributions}

AE initiated the project, designed the study, and was responsible for the concept. SE shared in the literature review, data collection, and writing the manuscript. AD did all the laboratory work. DE shared in manuscript writing study design, analysis of the results, and final revision of the manuscript. All authors provided critical feedback and helped shape and revise the research the analysis, and the manuscript. All authors have read and approved the manuscript.

\section{Funding}

The authors declare that no funds, grants, or other support were received.

Availability of data and materials

Data used to support the findings of this study are included within the article.

\section{Declarations}

\section{Ethics approval and consent to participate}

The ethical committee of the Faculty of Medicine, Alexandria University, approved this study. Written informed consent was obtained from both patients and the control. Committee reference No.: 0106135. Date: 24 September 2019. IRB No:: 00012098. FWA No: 00018699.

\section{Consent for publication}

Written informed consents were obtained from both patients and the control. The patients involved in this study agree to the publication of data.

\section{Competing interests}

The authors declare that they have no competing interests. 


\section{Author details}

${ }^{1}$ Tropical Medicine Department, Faculty of Medicine, Alexandria University, Alexandria, Egypt. ${ }^{2}$ Clinical Pathology Department, Faculty of Medicine, Alexandria University, Alexandria, Egypt.

Received: 4 March 2021 Accepted: 20 June 2021

Published online: 25 October 2021

\section{References}

1. Gill K, Ghazinian H, Manch R, Gish R (2016) Hepatitis C virus as a systemic disease: reaching beyond the liver. Hepatol Int 10(3):415-423

2. Crismale JF, Friedman SL (2020) Acute liver injury and decompensated cirrhosis. Med Clin North Am 104(4):647-662

3. D'Amico G, Morabito A, D'Amico M, Pasta L, Malizia G, Rebora P, Valsecchi MG (2018) Clinical states of cirrhosis and competing risks. J Hepatol 68(3):563-576

4. Tawfik N, El-Gendy N, Elhassan HAE, Ebrihem E, Saleh RA (2019) Inflammatory biomarkers as prognostic indicators for liver cirrhosis. Al-Azhar Assiut Med J 17(1):68-74

5. European Association for the Study of the Liver (EASL) (2018) EASL Clinical Practice Guidelines for the management of patients with decompensated cirrhosis. J Hepatol 69(2):406-460

6. Yokomizo T (2011) Leukotriene B4 receptors: novel roles in immunological regulations. Adv Enzyme Regul 51(1):59-64

7. Kalogeris T, Baines CP, Krenz M, Korthuis RJ (2012) Cell biology of ischemia/reperfusion injury. Int Rev Cell Mol Biol 298:229-317

8. Dirchwolf M, Podhorzer A, Marino M, Shulman C, Cartier M, Zunino M et al (2015) Immune dysfunction in cirrhosis: distinct cytokines phenotypes according to cirrhosis severity. Cytokine 77:14-25
9. Primdahl KG, Tungen JE, Aursnes M, Hansen TV Vik A (2015) An efficient total synthesis of leukotriene B4. Org Biomol Chem 13(19):5412-5417

10. Becares N, Maini A, Colas R, Dalli J, Gilroy D, O'Brien A (2019) FRI-109-Increased plasma leukotriene B4 in decompensated cirrhosis associates with disease progression and leads to increased skin window neutrophil infiltration. J Hepatol 70:e435

11. Asano F, Moriwaki H, Shiratori Y, Shimazaki M, Sakai T, Koshino Y et al (1993) Enhanced production of leukotriene B4 by peripheral blood mononuclear cells in patients with fulminant hepatitis. J Gastroenterol Hepatol 8(3):228-231

12. James $P$, Vajaravelu $S$, Radhakrishnan A (2016) Study of serum sodium levels in decompensated chronic liver disease. Glob J Res Anal 5(10):77-82

13. Lai C-Y, Cheng S-B, Lee T-Y, Liu H-T, Huang S-C, Huang Y-C (2018) Possible synergistic effects of glutathione and $\mathrm{C}$-reactive protein in the progression of liver cirrhosis. Nutrients 10(6):678

14. Ouwendijk RJ, Zijlstra FJ, Wilson JH, Vincent JE, Bonta IL (1985) Production of leukotrienes and prostaglandins by human ascites cells. Eur J Clin Invest 15(6):327-331

15. Moore KP, Taylor GW, Maltby NH, Siegers D, Fuller RW, Dollery CT, Williams R (1990) Increased production of cysteinyl leukotrienes in hepatorenal syndrome. J Hepatol 11(2):263-271

\section{Publisher's Note}

Springer Nature remains neutral with regard to jurisdictional claims in published maps and institutional affiliations.

\section{Submit your manuscript to a SpringerOpen ${ }^{\circ}$ journal and benefit from:}

- Convenient online submission

- Rigorous peer review

- Open access: articles freely available online

- High visibility within the field

Retaining the copyright to your article

Submit your next manuscript at $\boldsymbol{\nabla}$ springeropen.com 